\section{International Scientific Journal Theoretical \& Applied Science}

\author{
p-ISSN: 2308-4944 (print) e-ISSN: 2409-0085 (online) \\ Year: 2017 Issue: $07 \quad$ Volume: 51
}

Published: $13.07 .2017 \quad \underline{\text { http://T-Science.org }}$

SECTION 25. Technologies of materials for the light and textile industry.
Viktor Yureivich Mishakov doctor of technical Sciences, associate Professor Department of material science and commodity examination

Kosygin State University, Russia viktormishakov@ rambler.ru

Dimitry Anatolievich Sovetnikov graduate student Kosygin State University

Maksim Andreevich Pavlov graduate student Kosygin State University

Elena Aleksandrovna Kirsanova doctor of technical Sciences, Professor Kosygin State University

\title{
THEORETICAL METHOD ALLOWING CALCULATING AND ANALYZE THE EFFECTIVE COEFFICIENT OF THERMAL CONDUCTIVITY, NONWOVEN HEAT-SHIELDING MATERIALS
}

Abstract: Presented the method of analyzing and calculating the effective thermal conductivity of non-woven heat-insulating materials and experimental evaluation of a model of the temperature dependence of the effective thermal conductivity of insulation material for nonwoven products used in especially cold climatic zone.

Key words: glued, thermally bonded, needle-punched nonwoven cloth, bulk density, porosity (sponginess), dispersion environs, the fibrous canvas, the theoretical dependence material packets.

Language: Russian

Citation: Mishakov VY, Sovetnikov DA, Pavlov MA, Kirsanova EA (2017) THEORETICAL METHOD ALLOWING CALCULATING AND ANALYZE THE EFFECTIVE COEFFICIENT OF THERMAL CONDUCTIVITY, NONWOVEN HEAT-SHIELDING MATERIALS. ISJ Theoretical \& Applied Science, 07 (51): 21-27.

Soi: http://s-o-i.org/1.1/TAS-07-51-5 Doi: crossef https://dx.doi.org/10.15863/TAS.2017.07.51.5

\section{РАЗРАБОТКА МЕТОДА АНАЛИЗА И РАСЧЕТА ЭФФЕКТИВНОГО КОЭФФИЦИЕНТА ТЕПЛОПРОВОДНОСТИ НЕТКАНОГО ТЕПЛОИЗОЛЯЦИОННОГО МАТЕРИАЛА}

Аннотация: Представлен метод анализа и расчета эффективного коэффициента теплопроводности нетканых теплоизоляционных материалов и экспериментальная оценка модели температурной зависимости коэффициента эффективной теплопроводности нетканого теплоизоляционного материала для пакетов материалов изделий, применяемых в особо холодной климатической зоне.

Ключевые слова: клееные, термоскрепленные, иглопробивные нетканые полотна, объемная плотность, пористость, дисперсная среда, волокнистый холст, теоретическая зависимость эффективного коэффициента теплопроводности.

\section{Введение}

Ранее было выявлено, что существующие методы анализа и расчета эффективного коэффициента теплопроводности нетканого теплоизоляционного материала не учитывают условий эксплуатации, а также хаотическое расположение волокон в клееных, термоскрепленных, иглопробивных нетканых полотнах [1, с.87]. Система пакета теплозащитной одежды должна соответствовать размерам тела человека. обеспечить требования сохранения воздушной прослойки в пакете, и в тоже время создать необходимое прилегание к телу человека, в месте знакопеременной кривизны поверхности [2, с.150]. В утепленной верхней одежде покровная ткань, подкладочная ткань и утеплитель как правило состоят из материалов разной структуры: ткани, трикотажные и нетканые полотна $[3$, с.7; 4, с.60]. Комплект специальной одежды для военнослужащих пограничных органов можно использовать в различных физикогеографических 
условиях и в нем имеется два вида пакетов: Первый - в котором слои жестко соединены, то есть ни при каких условиях они не изменяют в своей конструкции. Изменение геометрических параметров таких пакетов, происходит только при изменении их толщины в определенных пределах, за счет упругих свойств утеплителя [5, .c.53; 6, с.19; 7, с.111] Второй тип - это пакеты, гибко соединённые с другими элементами одежды, которые могут быть выполнены или из других пакетов или из единичных материалов. В пакетах первого и второго типов и с лицевой и изнаночной стороны имеется ветростойкая прокладка, следовательно, передача тепла конвекцией практически будет отсутствовать, тепловое излучение материалов внутри пакета также останется постоянным поэтому возможно для оценки таких пакетов определять его теплоизолирующие свойства по показателю эффективного коэффициента теплопроводности [8, c 75,].

Основной теплоизолирующий материалнетканое полотно. В работе [9, с.34] отмечена некоторая неравномерность заполнения мононитями рассматриваемого микрообъема нетканого полотна и констатировано наличие плотной укладки и наличие пустот, а также отсутствие капилляров в общепринятом виде. В этой же работе предложено с целю физического моделирования нетканых полотен, рассматривать их как сплошную среду, имеющую пористое строение и волокнистую структуру.

Следует отметить, что волокнистые материалы относят к связнодисперсным системам с твердой поверхностью раздела фаз Г1/Т2 (по классификации Ребиндера индекс 1 обозначает дисперсную фазу, а индекс 2 дисперсионную среду) [10, с.6].

Установлено [11, с.35], что с увеличением объемной плотности дисперсного материала возрастает и коэффициент теплопроводности, это позволяет применить для анализа теплофизических свойств материала степенную зависимость:

$$
\square э \phi=\mathrm{L} \square \mathrm{m},
$$

где: $\square э ф, \quad \square \quad$ эффективный коэффициент теплопроводности,

$\square \square$ плотность материала соответственно,

$\mathrm{L} \square$ коэффициент пропорциональности,

$\mathrm{m} \square$ показатель, характеризующий степень нелинейности функции.

Конкретные величины плотностей разных материалов варьируются в широком диапазоне значений $(0<\square<\square)$ и точно задать верхний предел сложно, поэтому целесообразнее рассматривать следующую зависимость:

$$
\square_{\text {эф }}=L\left(\square_{\mathrm{M}} / \square_{\mathrm{B}}\right)^{\mathrm{m}},
$$

то есть

$$
\square_{b \phi}=F\left(\triangle_{\mathrm{M}} / \triangle_{\mathrm{B}}\right),
$$

где: $\square_{\mathrm{M}}, \square_{\mathrm{B}} \square$ плотности материала и структурных элементов соответственно.

Как правило в нетканых объемных утеплителях, объемная пористость материала близка к $100 \%$, следовательно величина $\square_{\mathrm{M}} /{ }_{\mathrm{B}} \square$ 0 , a для тонких материалов с пленочным покрытием, при минимально возможной пористости значение $\triangle \mathrm{M} / \triangle \mathrm{B}$ приближается к единице, т.е. $\triangle_{\mathrm{M}} \square \triangle_{\mathrm{B}}$.

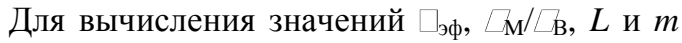
используется метод исследования на экстремум заданной функции при наличии дополнительных условий:

$$
F\left(\frac{\rho_{M}}{\rho_{B}}\right)= \begin{cases}0 & \text { nрu } \rho_{1} \leq \frac{\rho_{M}}{\rho_{B}} \leq \rho_{2} \\ \left(\frac{\rho_{M}}{\rho_{B}}-\rho_{2}\right) & \text { nрu } \frac{\rho_{M}}{\rho_{B}} \geq \rho_{2} \\ \left(\rho_{1}-\frac{\rho_{M}}{\rho_{B}}\right) & \text { nрu } \frac{\rho_{M}}{\rho_{B}} \leq \rho_{1}\end{cases}
$$

где: $\square_{1}, \quad \square_{2} \square$ величины отношения $\square \mathrm{M} /{ }_{\mathrm{B}}$ соответственно при минимальном $\square$ и максимальном ${ }_{2}$ значениях эффективного коэффициента теплопроводности.

$\square$ и $\square$ зависят от плотности волокон, толщины и пористости материала и среды, заполняющей его поровое пространство.

При максимальной пористости $(0 \square \square \mathrm{M} / \square \square$ $0,05)$ эффективный коэффициент теплопроводности материала практически равен коэффициенту теплопроводности среды, в данном случае воздуха, который при атмосферном давлении $P=1,01 \cdot 10^{5}$ Па и температуре $t{ }_{\text {воз }}=0{ }^{0} \mathrm{C}$ равен $\square_{\text {воз }}=0,02442$ $\mathrm{BT} /(\mathrm{M} \cdot \mathrm{K})$ [12, c.235].

Расчет численных значений $\square_{э ф}, \square_{\mathrm{M}} / \square_{\mathrm{B}}, L$ и $m$ и построение (рис. 1) зависимости $\square$ б $=F\left(\square \mathrm{M} / \triangle_{\mathrm{B}}\right)$ проводились при помощи комплекса «MATHCAD». В результате проведенных расчетов были определены искомые величины, позволяющие рассчитать оптимальное значение $\measuredangle \phi:$

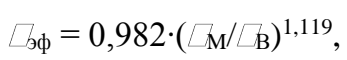

Следует отметить, что предложенный метод расчета требуемых значений эффективного коэффициента теплопроводности в зависимости от плотности дисперсной среды справедлив для всех материалов, имеющих капиллярнопористую или пористую структуру. 


\begin{tabular}{|c|c|c|c|c|c|c|}
\hline Impact Factor: & $\begin{array}{l}\text { ISRA (India) } \\
\text { ISI (Dubai, UAF } \\
\text { GIF (Australia) } \\
\text { JIF }\end{array}$ & $\begin{array}{l}=1.344 \\
=0.829 \\
=0.564 \\
=1.500\end{array}$ & $\begin{array}{l}\text { SIS (USA) } \\
\text { PИНЦ (Russia) } \\
\text { ESJI (KZ) } \\
\text { SJIF (Morocco) }\end{array}$ & $\begin{array}{l}=0.912 \\
=0.234 \\
=\mathbf{3 . 8 6 0} \\
=\mathbf{2 . 0 3 1}\end{array}$ & $\begin{array}{l}\text { ICV (Poland) } \\
\text { PIF (India) } \\
\text { IBI (India) }\end{array}$ & $\begin{array}{l}=6.630 \\
=1.940 \\
=4.260\end{array}$ \\
\hline
\end{tabular}

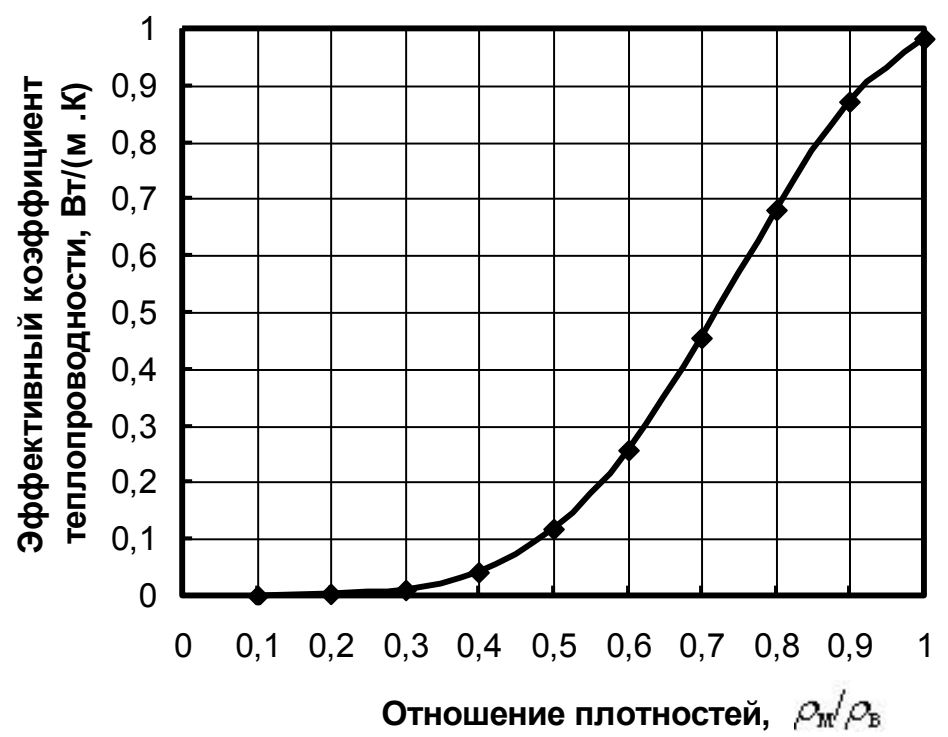

Рисунок 1 - Графическая интерпретация зависимости $\lambda_{\text {зф }}=F\left(\rho_{\mathrm{M}} / \rho_{\mathrm{B}}\right)$

Объекты и методы исследования. С целью оценки правомерности применения аналитических зависимостей для расчета эффективного коэффициента теплопроводности 乙ъф были проведены экспериментальные исследования нетканого полотна с помощью модифицированного измерителя ИТ- $\lambda-400$ в котором использован метод динамического калориметра (рис. 2),. Измеритель теплопроводности ИТ- $\lambda-400$ позволяет проводить испытания образцов в температурном интервале от $-50{ }^{0} \mathrm{C}$ до $200{ }^{0} \mathrm{C}$ с предельно допустимой погрешностью измерений не более $10 \%$.

Порядок выполнения измерений заключается в следующем. Испытуемый образец материала - 4, помещают на контактную пластину 3 тепломера и затем закрывают подвижной оболочкой 2, которая предохраняет измерительную ячейку от контакта с окружающей средой. После установки температуры, до которой необходимо нагреть образец, включают электронагреватель. Тепловой поток $Q(\square)$, направленный снизу вверх через основание 1 , нагревает образец до заданной температуры. Температура стержня 5 достигает ожидаемого значения при прохождении светового указателя прибора Ф136 через нулевую отметку.

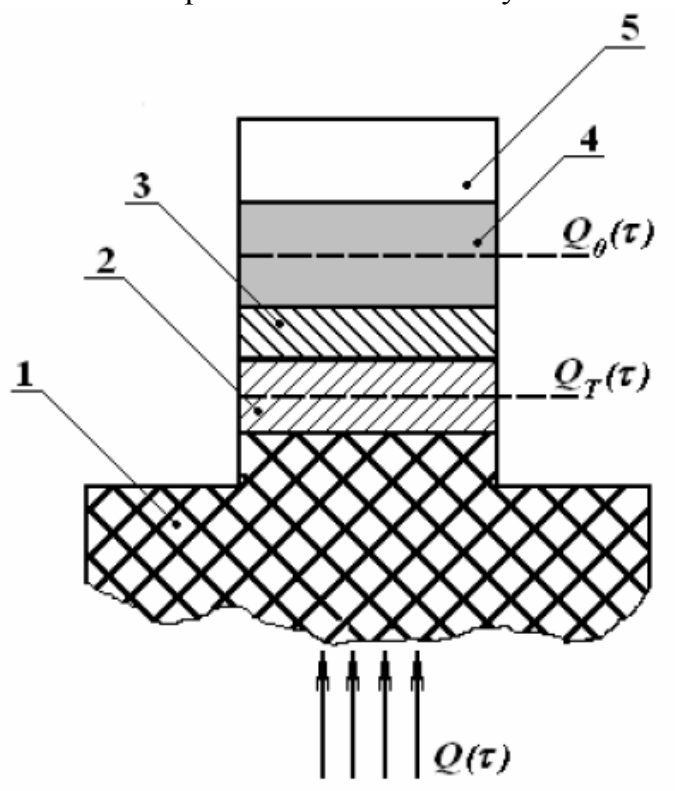

Рисунок 2 - Тепловая схема метода. 


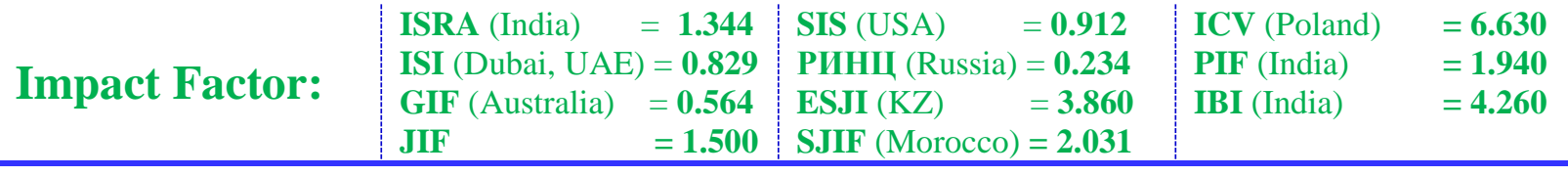

Переключая рукоятку переключателя «ИЗМЕРЕНИЕ» снимают показания прибора $\Phi 136 n_{\mathrm{o}}$ и $n_{\mathrm{T}}$ (в микроамперах).

После определения $n_{\mathrm{o}}$ и $n_{\mathrm{T}}$ устанавливают новое значение температуры и измерения повторяются.

Расчет коэффициента теплопроводности производят в следующей последовательности.

Поправка на теплоемкость образца:

$$
\square_{\mathrm{c}}=C_{\mathrm{o}} /\left[2\left(C_{\mathrm{o}}+C_{\mathrm{c}}\right)\right] \text {, }
$$

где: $C_{\mathrm{o}}-$ полная теплоемкость образца, Дж/К;

$C_{\mathrm{c}}(t)$ - полная теплоемкость стержня 5(по паспорту установки), Дж/К;

Тепловое сопротивление:

$$
R_{\mathrm{o}}=\left[\square_{\mathrm{o}} S \cdot(1+\square \mathrm{c}) /\left(\square_{\mathrm{T}} K_{\mathrm{T}}\right)\right]-\square R_{\mathrm{K}},
$$

где: $\square_{6}$ - перепад температур на образце, соответствующий значению $n_{\mathrm{o}}$;

$\square_{\text {r }}$ - перепад температур на пластине, соответствующий значению $n_{\mathrm{T}}$;

$S$ - площадь поперечного сечения образца, $\mathbf{m}^{2}$;

$K_{\mathrm{T}}(t) \quad-\quad$ коэффициент, характеризующий теплопроводность пластины, Вт/К;

$\square R_{\text {к }}(t)$ - поправка, учитывающая сопротивление контакта, не идентичность и тепловое сопротивление заделки термопар, $\mathrm{M}^{2} \cdot \mathrm{K} / \mathrm{B}$.

Значения $K_{\mathrm{T}}(t)$ и $\square R_{\mathrm{K}}(t)$, также как $C_{\mathrm{c}}(t)$ заданы в паспортных данных установки.

Величина коэффициента теплопроводности образца:

$$
\square=h / R_{\mathrm{o}},
$$

где: $h$ - толщина исследуемого образца, м.

Для экспериментальной проверки использовали нетканый стекловолокнистый холст марки ХСБТ-90 (ТУ 6-48-00204949-92) Судогдского завода теплоизоляционных изделий объемной плотностью $\nabla_{\mathrm{M}}=51,4$ кг $/ \mathrm{M}^{3}$. В качестве объектов исследования также были выбраны пакеты материалов для одежды, используемой в арктической зоне

Пакет № 1 - куртка (верх) - ткань полиэфирная ветровлагозащитная гладкокрашеная арт. ПСЗО-1/2, прокладка-ткань ветрозащитная арт. НТУТ-5/2M, утеплитель «РМ-200» 2 слоя, подкладка - ткань подкладочная полиэфирная с антистатической отделкой арт. НТУТ-5/4М; - полукомбинезон (верх) - ткань полиэфирная ветровлагозащитная гладкокрашеная арт. ПСЗО-1/2, утеплитель «РМ-200» 1 слой, подкладка - ткань подкладочная полиэфирная с антистатической отделкой арт. НТУТ-5/4М; - жилет (верх) - ткань полиэфирная ветровлагозащитная арт. ПСЗО-1/2 гладкокрашеная, утеплитель - «РМ-200» 1 слой, подкладка - ткань подкладочная полиэфирная с антистатической отделкой арт. НТУТ-5/4М.

Пакет № 2 - куртка (верх) - ткань полиэфирная ветровлагозащитная гладкокрашеная арт. ПСЗО-1/2, прокладка-ткань ветрозащитная арт. НТУТ-5/2M, утеплитель «РМ-150» 2 слоя, подкладка - ткань подкладочная полиэфирная с антистатической отделкой арт. НТУТ-5/4М; - полукомбинезон (верх) - ткань полиэфирная ветровлагозащитная гладкокрашеная арт. ПСЗО-1/2, утеплитель «РМ-150» 1 слой, подкладка - ткань подкладочная полиэфирная с антистатической отделкой арт. НТУТ-5/4М; - жилет (верх) - ткань полиэфирная ветровлагозащитная арт. ПСЗО-1/2 гладкокрашеная, утеплитель - «РМ-150» 1 слой, подкладка - ткань подкладочная полиэфирная с антистатической отделкой арт. НТУТ-5/4М. [11,.c.35; 13, с.158].

Перепад температур в образцах составлял 10 ${ }^{0} \mathrm{C}$, атмосферное давление $P=1,01 \cdot 10^{5}$ Па, относительная погрешность измерений коэффициента теплопроводности не превышала $2,9 \%$.

Результаты и обсуждение.

Проведены эксперименты, позволяющие определить значения коэффициента пропорциональности $(L)$ и показателя степени нелинейности функции $(m)$ при заданных

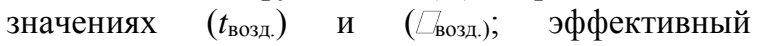
коэффициент теплопроводности ( $\left.{ }_{g \phi}\right)$ и одновременно теоретически рассчитан эффективный коэффициент теплопроводности по формуле $\square_{\text {фф }}=0,982 \cdot\left(\triangle_{\mathrm{M}} / \triangle_{\mathrm{B}}\right)^{1,119}$. (см. табл.1.).

\begin{tabular}{|c|c|c|c|c|c|c|}
\hline \multicolumn{4}{|c|}{$\begin{array}{c}\text { Расчетные значения коэффициента } \\
\text { пропорциональности }(L) \text { и показателя степени } \\
\text { нелинейности функции }(m) \text { при заданных } \\
\text { значениях } t_{\text {возд }} \text { и } \square_{\text {возд }}\end{array}$} & \multicolumn{2}{|c|}{$\begin{array}{c}\text { Эффективный коэффициент } \\
\text { теплопроводности } \square_{э ф}, \text { Вт/(м·К) }\end{array}$} & \multirow{2}{*}{$\begin{array}{c}\text { Относительная } \\
\text { погрешность } \\
\square, \square\end{array}$} \\
\hline $\begin{array}{l}\text { температура } \\
\text { воздуха, } \\
t_{\text {возд, }}{ }^{0} \mathrm{C}\end{array}$ & $\begin{array}{l}\text { коэффициент } \\
\text { теплопроводности } \\
\text { воздуха, } \\
\quad \square_{\text {возд }}, \mathrm{BT} /(\mathrm{M} \cdot \mathrm{K})\end{array}$ & $L$ & $m$ & $\begin{array}{l}\text { расчетное } \\
\text { значение }\end{array}$ & $\begin{array}{c}\text { экспериментальное } \\
\text { значение }\end{array}$ & \\
\hline
\end{tabular}

\section{Таблица 1}

Расчетные данные для определения эффективного коэффициента теплопроводности. 


\begin{tabular}{|c|c|c|c|c|c|c|}
\hline Impact Factor: & $\begin{array}{l}\text { ISRA (India) } \\
\text { ISI (Dubai, UAE } \\
\text { GIF (Australia) } \\
\text { JIF }\end{array}$ & $\begin{array}{l}=1.344 \\
=0.829 \\
=0.564 \\
=1.500\end{array}$ & $\begin{array}{l}\text { SIS (USA) } \\
\text { PИНЦ (Russia) } \\
\text { ESJI (KZ) } \\
\text { SJIF (Morocco }\end{array}$ & $\begin{array}{l}=0.912 \\
=\mathbf{0 . 2 3 4} \\
=\mathbf{3 . 8 6 0} \\
=\mathbf{2 . 0 3 1}\end{array}$ & $\begin{array}{l}\text { ICV (Poland) } \\
\text { PIF (India) } \\
\text { IBI (India) }\end{array}$ & $\begin{array}{l}=6.630 \\
=1.940 \\
=4.260\end{array}$ \\
\hline
\end{tabular}

\begin{tabular}{|c||c||c||c||c||c||c|}
\hline-30 & 0,022 & 0,992 & 1,148 & 0,03924 & 0,0373 & $-5,2$ \\
\hline \hline-20 & 0,0228 & 0,988 & 1,139 & 0,04009 & 0,0380 & $-5,5$ \\
\hline \hline-10 & 0,0236 & 0,987 & 1,129 & 0,04119 & 0,0382 & $-7,8$ \\
\hline \hline 0 & 0,0244 & 0,982 & 1,119 & 0,04216 & 0,0389 & $-8,3$ \\
\hline \hline 10 & 0,0251 & 0,980 & 1,116 & 0,04243 & 0,0395 & $-7,4$ \\
\hline \hline 20 & 0,0259 & 0,977 & 1,113 & 0,04266 & 0,0410 & $-4,0$ \\
\hline \hline 30 & 0,0267 & 0,976 & 1,109 & 0,04309 & 0,0420 & $-2,6$ \\
\hline
\end{tabular}

С целью подтверждения адекватности теоретической модели расчета эффективного коэффициента теплопроводности выполнена оценка относительной погрешности вычислений $(\square)$ :

$$
\varepsilon=\frac{\left(\lambda_{\ni}-\lambda_{T}\right)}{\lambda_{\ni}} \cdot 100, \%
$$

где: $\square_{b}, \square_{\mathrm{T}}-$ экспериментальные и расчетные (теоретические) значения эффективного коэффициента теплопроводности соответственно.

Отклонения результатов (табл. 1) теоретических от экспериментальных данных находятся в пределах от $-2,6$ \% до $-8,3 \%$, что не превышает предельно допустимую погрешность измерений, и полностью подтверждает адекватность разработанной модели.

Полученные данные были обработаны с использованием программы Microsoft Excel, аппроксимация теоретических зависимостей эффективных коэффициентов теплопроводности от температуры среды показала линейный характер (рис. 3) с высокой теснотой связей результативного признака с исследуемым фактором:

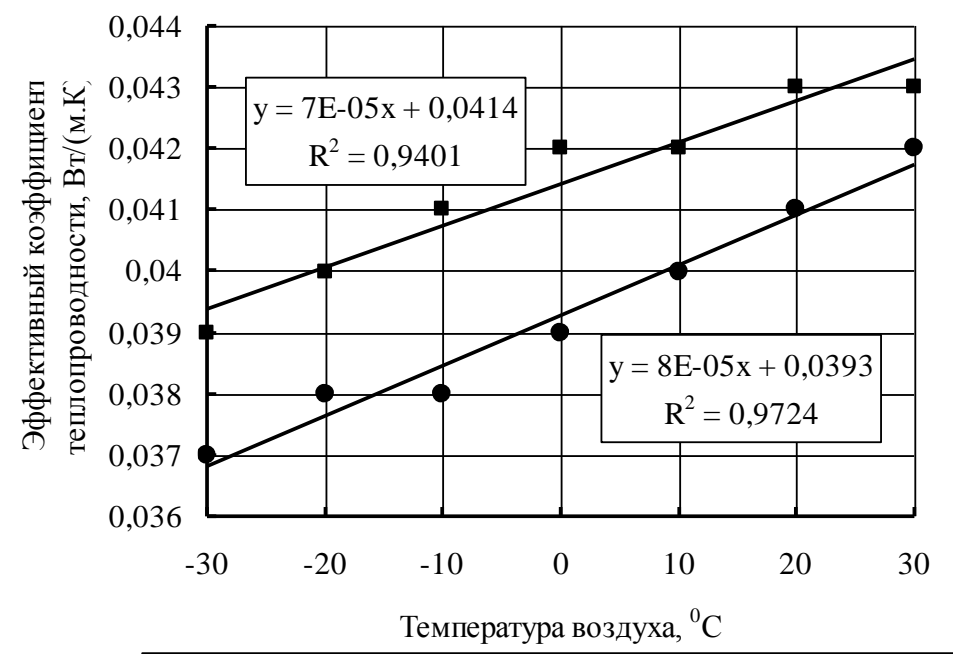

- экспериментальные значения - теоретические значения

\section{Рисунок 4 - Температурная зависимость коэффициента эффективной теплопроводности волокнистого холста.}

Отклонения теоретических результатов от экспериментальных данных находятся в пределах от 2,6 \% до 8,3 \%, что не превышает предельно допустимую погрешность измерений, и полностью подтверждает адекватность разработанной модели. Таким образом, полученные модели регрессии температурной зависимости коэффициента эффективной теплопроводности, хорошо аппроксимируют исходные данные и такой регрессионной моделью можно воспользоваться для прогнозирования теплофизических свойств нетканого волокнистого полотна.

Для оценки теплосохраняющих свойств утепленного костюма были определены коэффициента теплопроводности пакетов материалов (см.таб.2) 


\begin{tabular}{|c|c|c|c|c|c|c|}
\hline Impact Factor: & $\begin{array}{l}\text { ISRA (India) } \\
\text { ISI (Dubai, UAF } \\
\text { GIF (Australia) } \\
\text { JIF }\end{array}$ & $\begin{array}{l}=1.344 \\
=0.829 \\
=0.564 \\
=1.500\end{array}$ & $\begin{array}{l}\text { SIS (USA) } \\
\text { PИНЦ (Russia) } \\
\text { ESJI (KZ) } \\
\text { SJIF (Morocco) }\end{array}$ & $\begin{array}{l}=0.912 \\
=0.234 \\
=3.860 \\
=\mathbf{2 . 0 3 1}\end{array}$ & $\begin{array}{l}\text { ICV (Poland) } \\
\text { PIF (India) } \\
\text { IBI (India) }\end{array}$ & $\begin{array}{l}=6.630 \\
=1.940 \\
=4.260\end{array}$ \\
\hline
\end{tabular}

Таблица 2

Тепловое сопротивление отдельных элементов верхней одежды для арктической зоны.

\begin{tabular}{|c|c|c|c|c|c|c|c|}
\hline $\begin{array}{c}\text { Наименование } \\
\text { элементов } \\
\text { комплекта } \\
\text { одежды }\end{array}$ & $\begin{array}{c}\text { Поверхнос } \\
\text { тная } \\
\text { плотность, } \\
\text { г/м }^{2}\end{array}$ & $\begin{array}{l}\text { Толщина } \\
\text { при } \\
\text { давлении } \\
0,5 \text { кПа, } h, \\
\text { мм }\end{array}$ & $\begin{array}{c}\text { Суммарно } \\
\text { е тепловое } \\
\text { сопротивл } \\
\text { ение, } R_{0}, \\
\mathrm{M}^{2} \cdot \mathrm{K} / \mathrm{BT}\end{array}$ & $\begin{array}{c}\text { Темп } \\
\text { охлаждени } \\
\text { я, с }\end{array}$ & $\begin{array}{c}\text { Пористость, } \\
П, \%\end{array}$ & $\mid \begin{array}{c}\text { Объем. } \\
\text { плотность } \\
\cdot \rho_{\mathrm{M}}, \Gamma / \mathrm{M}^{3}\end{array}$ & $\begin{array}{c}\text { Коэффици } \\
\text { ент } \\
\text { теплопров } \\
\text { одности } \lambda \text {, } \\
\text { Вт/(м·К) }\end{array}$ \\
\hline $\begin{array}{c}\text { Пакет № } 1 \\
\text { с утеплителем } \\
\text { РМ-400 }\end{array}$ & 2100 & 63,2 & 0,74 & 1003 & 96,9 & 42,38 & 0,035 \\
\hline $\begin{array}{c}\text { Пакет № } 1 \\
\text { с утеплителем } \\
\text { РМ-350 } \\
\end{array}$ & 2050 & 61,3 & 0,72 & 1185 & 96,8 & 43,52 & 0,035 \\
\hline $\begin{array}{c}\text { Пакет № } 1 \\
\text { с утеплителем } \\
\text { РМ-300 }\end{array}$ & 2000 & 60,2 & 0,70 & 1080 & 95,6 & 60,25 & 0,038 \\
\hline $\begin{array}{c}\text { Пакет № } 1 \\
\text { с утеплителем } \\
\text { РМ-250 }\end{array}$ & 1980 & 58,0 & 0,69 & 948 & 94,3 & 79,16 & 0,042 \\
\hline $\begin{array}{c}\text { Пакет № } 1 \\
\text { с утеплителем } \\
\text { РМ-200 } \\
\end{array}$ & 1979 & 57,3 & 0,68 & 1145 & 94,1 & 81,20 & 0,043 \\
\hline $\begin{array}{c}\text { Пакет № } 1 \\
\text { с утеплителем } \\
\text { РМ-150 } \\
\end{array}$ & 1882 & 39 & 0,67 & 1026 & 93,8 & 85,55 & 0,043 \\
\hline $\begin{array}{c}\text { Пакет 1 } \\
\text { с утеплителем } \\
\text { РМ-100 }\end{array}$ & 1800 & 38,0 & 0,56 & 807 & 92,8 & 99,11 & 0,046 \\
\hline
\end{tabular}

Как видно из полученных результатов основное значение для теплосохраняющей способности иглопробивных нетканых утеплителей имеет объемная плотность материала, так, с ее повышением адекватно увеличивается эффективный коэффициент теплопроводности. Численный анализ, позволил установить следующие математические зависимости:

$\square=0,0002 \rho_{\mathrm{M}}+0,0263$, достоверность аппроксимации $\mathrm{R}^{2}=0,9999$;

$\square=-0,2856 \Pi+0,3118$, достоверность аппроксимации $\mathrm{R}^{2}=0,9997$.

На основании полученных данных произведены расчеты необходимой толщины пакетов теплозащитных изделий для разного температурного диапазона

Таким образом, полученные модели регрессии температурной зависимости коэффициента эффективной теплопроводности, хорошо аппроксимируют исходные данные и такой регрессионной моделью можно воспользоваться для прогнозирования теплофизических свойств нетканого волокнистого полотна.

\section{Выводы}

Предложенный метод расчета требуемых значений эффективного коэффициента теплопроводности в зависимости от плотности дисперсной среды справедлив для всех материалов, имеющих капиллярно-пористую или пористую структуру.

Предложена модель прогнозирования теплофизических свойств и толщины нетканого волокнистого полотна на основе экспериментально полученных регрессионных моделей температурной зависимости эффективного коэффициента теплопроводности, расширяющая границы применимости полученных результатов. Полученные теоретические модели использованы для определения толщины утеплителей для применения в защитной одежде при температурах окружающего воздуха до - $50^{\circ} \mathrm{C}$ 


\begin{tabular}{l|lrl|l|ll} 
& ISRA (India) & $=\mathbf{1 . 3 4 4}$ & SIS (USA) & $=\mathbf{0 . 9 1 2}$ & ICV (Poland) & $=\mathbf{6 . 6 3 0}$ \\
Impact Factor: & ISI (Dubai, UAE) $=\mathbf{0 . 8 2 9}$ & PUHU (Russia) $=\mathbf{0 . 2 3 4}$ & PIF (India) & $=\mathbf{1 . 9 4 0}$ \\
& GIF (Australia) & $\mathbf{0 . 5 6 4}$ & ESJI (KZ) & $=\mathbf{3 . 8 6 0}$ & IBI (India) & $=\mathbf{4 . 2 6 0}$ \\
& JIF & $=\mathbf{1 . 5 0 0}$ & SJIF (Morocco) & $=\mathbf{2 . 0 3 1}$ & & \\
\hline
\end{tabular}

\section{References:}

1. Sovetnikov DA, Mishakov VY, Pavlov MA, Kirsanova EA, Treshchalin YM (2017) Teoreticheskoe issledovanie voloknistyh materialov s cel'yu rascheta i prognozirovaniya teplofizicheskih svojstv //Dizajn i tekhnologii. 2017. № 57 (99). p. 86-91.

2. Sovetnikov DA, Mishakov VY, Kirsanova EA (2017) The study of deformation characteristics of heaters and packages of clothing for military personnel that apply in the arctic zone of the russian federation. ISJ Theoretical \& Applied Science, 04 (48): 145-150. Doi: https://dx.doi.org/10.15863/TAS.2017.04.48.23

3. Sovetnikov DA, Mishakov VY, Zhagrina I.N., Muhamedzhanov G.K. Assortiment i oblasti primeneniya sinteticheskih uteplitelej// Dizajn i tekhnologii. 2013. № 34 (76). p. 62-69.

4. Surzhenko EY, Belgorodskiy VS (2012) Razrabotka informatsionno-logicheskoy modeli protsessa proyektirovaniya izdeliy $\mathrm{v}$ strukture ergonomicheskogo dizayna spetsialnoy odezhdy//Dizayn i tekhnologii. 2012. № 32 (74). p. 56-62.

5. Mishakov VY, Makarova NA, Stalevich AM (2004) Relaxation and delay times in nonwoven fabrics. //Izvestiya vysshikh uchebnykh zavedeniy. Tekhnologiya tekstilnoy promyshlennosti. 2004. № 4. p. 52-55.

6. Mishakov VY, Zheleznyakov AS (2004) Eksperimentalnoye issledovaniye NDS myagkikh kompozitov posredstvm mekhanicheskikh kolebaniy..// Materialovedeniye. 2004. № 10. p. 19.

7. Kiselev MV, Belgorodskiy VS, Smirnova NA (2012) Obosnovaniye ekonomicheskoy tselesoobraznosti matematicheskogo modelirovaniya uteplyayushchikh netkanykh poloten dlya shveynykh izdeliy spetsialnogo naznacheniya//Finansovaya ekonomika. 2012. № 6. p. 109-112.

8. Sovetnikov DA, Mishakov VY, Kirsanova EA, Treshchalin YM (2016) Issledovanie teplozashchitnyh svojstv netkanyh uteplitelej v paketah odezhdy [Tekst]//Dizajn i tekhnologii. 2016. № 56 (98). p.73-79.

9. Treshchalin YM, Kiselev MV, Khammatova VV, Treshchalin MY, Kiselev AM (2015) Issledovaniye struktury netkanykh materialov metodom kompyuternoy tomog rafii //Izv. vuzov. Tekhnologiya tekstilnoy promyshlennosti. 2015. No5 (359). p.31-34.

10. (2005) Poverkhnostnyye yavleniya v dispersnykh sistemakh: Ucheb. posobiye/L.V. Koltsov. M.A. Loseva; Samar. gos. tekhn. un-t. Samara. 2005. 140 p.

11. Tyumenev YY, Treshchalina AV, Nazarova YV, Treshchalin MY (2009) sovershenstvovaniye metodov rascheta i otsenki teploizolyatsionnykh svoystv netkanykh materialov/l VESTNIK ASSOTsIATsII VUZOV TURIZMA I SERVISA .Rossiyskiy gosudarstvennyy universitet turizma i servisa 2009.» №4, p.33-39.

12. Dulnev GN, Zarichnyak YP (1974) Teploprovodnost smesey i kompozitsionnyy materialov. L.:Energiya. 1974. 264 p.

13. Mishakov VY (2017) Razvitiye nauchnometodicheskikh osnov razrabotki antimikrobnykh i zashchitnykh materialov na netkanykh voloknistykh nositelyakh i metodov ikh issledovaniya. Monografiya. Moskva. 2017. 207 p. 